\title{
Rate of homogeneous crystal nucleation in molten $\mathrm{NaCl}$
}

\author{
C. Valeriani ${ }^{\mathrm{a})}$ \\ Fundamenteel Onderzoek der Materie (FOM), Institute for Atomic and Molecular Physics, Kruislaan 407, \\ 1098 SJ Amsterdam, The Netherlands \\ E. Sanz \\ Facultad de Ciencias Químicas, Universidad Complutense de Madrid, 28040 Madrid, Spain \\ D. Frenkel \\ Fundamental Onderzoek der Materie (FOM), Institute for Atomic and Molecular Physics, Kruislaan 407, \\ 1098 SJ Amsterdam, The Netherlands
}

(Received 7 September 2004; accepted 28 February 2005; published online 13 May 2005)

\begin{abstract}
We report a numerical simulation of the rate of crystal nucleation of sodium chloride from its melt at moderate supercooling. In this regime nucleation is too slow to be studied with "brute force" molecular-dynamics simulations. The melting temperature of ("Tosi Fumi") $\mathrm{NaCl}$ is $\sim 1060 \mathrm{~K}$. We studied crystal nucleation at $T=800$ and $825 \mathrm{~K}$. We observe that the critical nucleus formed during the nucleation process has the crystal structure of bulk $\mathrm{NaCl}$. Interestingly, the critical nucleus is clearly faceted, the nuclei have a cubical shape. We have computed the crystal-nucleation rate using two completely different approaches, one based on an estimate of the rate of diffusive crossing of the nucleation barrier, the other based on the forward flux sampling and transition interface sampling methods. We find that the two methods yield the same result within an order of magnitude. However, when we compare the extrapolated simulation data with the only available experimental results for $\mathrm{NaCl}$ nucleation, we observe a discrepancy of nearly five orders of magnitude. We discuss the possible causes for this discrepancy. (C) 2005 American Institute of Physics.
\end{abstract}

[DOI: $10.1063 / 1.1896348$ ]

\section{INTRODUCTION}

Crystallization of salts is a phenomenon of great practical relevance. In fact, it is one of the most important industrial separation processes. But it also plays a crucial role in geological processes that occur on an altogether different time scale. The crystallization process consists of two steps: nucleation and growth. If nucleation is slow compared to the time it takes a crystal to grow to a size comparable to the size of the container, large single crystals will form (an example is rock salt). When nucleation is fast, the resulting solid will form as a fine powder. It is clearly important to be able to predict the rate of nucleation of salts and-at a later stage - to understand the factors that influence nucleation. In the present paper we aim to demonstrate that, with current simulation techniques and currently available force fields, it is indeed possible to compute the rate of nucleation of a real salt crystal (in the present case $\mathrm{NaCl}$ from its melt). This opens the way to $a b$ initio predictions of nucleation rates of many ionic substances.

Solutions or melts can often be cooled well below their freezing temperature. The reason is that the formation of small nuclei of the stable crystal phase is an activated process that may be extremely slow. Intuitively, it is easy to understand why crystal nucleation is an activated process, i.e., why there is a free-energy barrier separating the metastable parent phase (the liquid) from the stable crystal phase. The point is that, initially, the formation of small crystalline

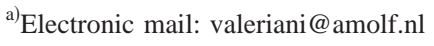

nuclei costs free energy. But once the crystal nucleus exceeds a critical size, its free energy decreases as it grows. The rate at which crystal nuclei form depends strongly on $\Delta G_{\text {crit }}$, the free energy required to form a critical nucleus. Classical nucleation theory (CNT) is commonly used to estimate the height of the nucleation barrier and to predict the rate of crystal nucleation. ${ }^{1-5}$ According to CNT, the total free energy of a crystallite that forms in a supersaturated solution or melt contains two terms: the first is a "bulk" term that expresses the fact that the solid is more stable than the supersaturated fluid-this term is negative and proportional to the volume of the crystallite. The second is a "surface" term that takes into account the free-energy cost of creating a solid-liquid interface. This term is positive and proportional to the surface area of the crystallite. According to CNT, the total (Gibbs) free-energy cost to form a spherical crystallite with radius $R$ is

$$
\Delta G=\frac{4 \pi}{3} R^{3} \rho_{S} \Delta \mu+4 \pi R^{2} \gamma,
$$

where $\rho_{S}$ is the number density of the solid, $\Delta \mu(<0)$ the difference in chemical potential of the solid and the liquid, and $\gamma$ is the solid-liquid interfacial free-energy density. The function $\Delta G$ goes through a maximum at $R=2 \gamma /\left(\rho_{S}|\Delta \mu|\right)$ and the height of the nucleation barrier is 


$$
\Delta G_{\text {crit }}=\frac{16 \pi}{3} \gamma^{3} /\left(\rho_{S}|\Delta \mu|\right)^{2}
$$

The crystal-nucleation rate per unit volume, $I$, depends strongly on $\Delta G_{\text {crit }}$ :

$$
I=\kappa \exp \left(-\Delta G_{\text {crit }} / k_{B} T\right) .
$$

Here $\kappa$ is a kinetic prefactor, $T$ is the absolute temperature, and $k_{B}$ is Boltzmann's constant. The CNT expression for the nucleation rate then becomes

$$
I=\kappa \exp \left[-\frac{16 \pi}{3} \gamma^{3} /\left(\rho_{S}|\Delta \mu|\right)^{2}\right] .
$$

Under experimental conditions, nucleation is infrequent on the time scale of typical molecular processes. Yet, when it happens, it proceeds rapidly. This makes it difficult to study the structure and dynamics of crystal nuclei of atoms or small molecules in experiments. In the case of $\mathrm{NaCl}$, the experiments are also complicated by the fact that crystallization occurs at high temperatures. This may explain why there is a scarcity of experimental data on the nucleation of $\mathrm{NaCl}$. To our knowledge, the only data are those of Buckle and Ubbelohde $^{6}$ and Buckle $^{7}$ from the 1960s. In these experiments, crystallization in $\mathrm{NaCl}$ microdroplets was observed visually. As the droplet size $[\mathcal{O}(3 \mu \mathrm{m})]$ and time window for the measurement $(1-30 \mathrm{~s}-$ after which the droplets sedimented out of view) - were fixed, the nucleation rate could be determined at one temperature only $(905 \mathrm{~K}$ for $\mathrm{NaCl})$. At this temperature, the nucleation rate was such that, on average, one nucleus would form during the observation time $[\mathcal{O}(10 \mathrm{~s})]$ in a droplet with a volume of order $10^{-17} \mathrm{~m}^{3}$. Hence the experimental nucleation rate per unit volume was $O\left(10^{16}\right) \mathrm{m}^{-3} \mathrm{~s}^{-1}$.

For experimental nucleation rates of this order of magnitude, brute-force molecular-dynamics (MD) simulations are out of the question. The average time it would take for nuclei to form spontaneously in a system consisting of several thousands of particles is of the order of $10^{20} \mathrm{~s}$. Clearly, this is beyond the scope of MD simulations. The standard solution to circumvent this problem is to perform simulations at much larger undercooling than used in the experiments. Huang et $a l^{8}{ }^{8}$ performed MD simulations of melting and freezing of a droplet composed of $216 \mathrm{NaCl}$ ions in vacuum; to this end, they performed temperature quenches down to $550 \mathrm{~K}$ (i.e., approximately half the melting temperature) and found nucleation rates of the order of $O\left(10^{36}\right) \mathrm{m}^{-3} \mathrm{~s}^{-1}$, which is 20 orders of magnitude higher than the experimental rate at $905 \mathrm{~K}$.

Another effort to study nucleation at less severe supercooling was made by Koishi $e t$ al. ${ }^{9}$ These authors performed a MD simulation of 125000 ions system in vacuum, at temperatures of $740 \mathrm{~K}$ (i.e., approximately 0.7 the melting temperature $T_{m}$ ) and $640 \mathrm{~K}$ (i.e., approximately 0.6 the melting temperature $T_{m}$ ). Both free and periodic boundary conditions were used. The estimated nucleation rate at $740 \mathrm{~K}$ was $O\left(10^{35}\right) \mathrm{m}^{-3} \mathrm{~s}^{-1}$, which is virtually the same value as found by Huang et al. ${ }^{8}$ at a much larger supercooling. This is surprising because nucleation rates tend to depend very strongly on temperature. This suggests that, at least at the lowest tem-
TABLE I. Potential parameters for $\mathrm{NaCl}$.

\begin{tabular}{lcccrr}
\hline \hline & $\begin{array}{c}A_{i j} \\
(\mathrm{~kJ} / \mathrm{mol})\end{array}$ & $\begin{array}{c}B \\
\left(\AA^{-1}\right)\end{array}$ & $\begin{array}{c}C_{i j} \\
\left(\AA^{6} \mathrm{~kJ} / \mathrm{mol}\right)\end{array}$ & $\begin{array}{c}D_{i j} \\
\left(\AA^{8} \mathrm{~kJ} / \mathrm{mol}\right)\end{array}$ & \multicolumn{1}{c}{$\begin{array}{c}\sigma_{i j} \\
(\AA)\end{array}$} \\
\hline $\mathrm{Na}-\mathrm{Na}$ & 25.4435 & 3.1546 & 101.1719 & 48.1771 & 2.340 \\
$\mathrm{Na}-\mathrm{Cl}$ & 20.3548 & 3.1546 & 674.4793 & 837.0770 & 2.755 \\
$\mathrm{Cl}-\mathrm{Cl}$ & 15.2661 & 3.1546 & 6985.6786 & 14031.5785 & 3.170 \\
\hline \hline
\end{tabular}

peratures, the barrier for crystal nucleation is negligible. More in general, crystal nucleation under extreme supercooling does not need to proceed following the same path as under moderate supercooling. ${ }^{5}$

In what follows, we use the technique of Refs. 10-12 based on a combination of umbrella sampling ${ }^{13}$ (to determine the barrier height) and a dynamical simulation (to determine the crossing rate). The computing time required for this scheme does not scale exponentially with the nucleation barrier, but it does increase with increasing nucleus size.

We also compute the nucleation rate using an algorithm based on the "forward flux sampling"14 (FFS) and the "transition interface sampling", (TIS) techniques and compare it with the one obtained using the method previously mentioned.

In the present work we study homogeneous crystal nucleation in the Tosi-Fumi $\mathrm{NaCl}$ model for $\mathrm{NaCl}$ at two different temperatures, viz., $T_{1}=800 \mathrm{~K}$ and $T_{2}=825 \mathrm{~K}$, corresponding to $25 \%$ and $22 \%$ undercooling. For this system, we computed the nucleation barrier, examined the structure and shape of the critical nucleus, and computed the nucleation rate.

\section{METHODS}

The Tosi-Fumi rigid-ion interaction potential for $\mathrm{NaCl}$ is of the following form: ${ }^{16}$

$$
U_{i j}(r)=A_{i j} e^{\left[B\left(\sigma_{i j}-r\right)\right]}-\frac{C_{i j}}{r^{6}}-\frac{D_{i j}}{r^{8}}+\frac{q_{i} q_{j}}{4 \pi \epsilon r},
$$

where the parameters have the values given in Table I.

This pair potential is written as the sum of a BornHuggins-Mayer repulsion, two attractive van der Waals contributions, and a Coulomb interaction term. In our simulations, we calculated the Coulomb interactions using the Ewald summations method with a real-space cutoff of $10 \AA$ and a real-space damping parameter of $0.25 \AA^{-1}$. We truncated the van der Waals part of the potential at $9 \AA$, and we added tail corrections assuming the $g(r)=1$ beyond this cutoff.

TABLE II. Surface free-energy density (in erg $\mathrm{cm}^{-2}$ ) assuming spherical and cubical shapes for critical nuclei. At $905 \mathrm{~K}$ we report the experimental value (see Ref. 6). The entry in the lower right-hand corner is based on the experimental estimate, but assuming a cubical nucleus.

\begin{tabular}{ccc}
\hline \hline$T(\mathrm{~K})$ & $\gamma_{\text {sphere }}$ & $\gamma_{\text {cube }}$ \\
\hline 800 & $98 \pm 2$ & $80 \pm 1$ \\
825 & $99 \pm 1$ & $79 \pm 1$ \\
905 & $84.1^{(e)}$ & $67.8^{(e)}$ \\
\hline \hline
\end{tabular}


The computed number density of ions in the bulk solid at 800 and $825 \mathrm{~K}$ at $10^{5} \mathrm{~Pa}$ was $0.041 \AA^{-3}$, in agreement with the experiment. ${ }^{17}$ The density of ions in the supercooled liquid at the same temperature and pressure was $0.034 \AA^{-3}$.

We prepared under cubic boundary conditions a supercooled system of $(12)^{3} \mathrm{NaCl}$ ion pairs at ambient pressure by cooling it down below the melting temperature. For the present model, Anwar et al. ${ }^{18}$ have computed the melting temperature: $T_{m}=1064 \pm 14 \mathrm{~K}$, which is very close to the experimental melting temperature $\left(T_{m}^{\exp }=1072 \mathrm{~K}\right)$. Using constant-pressure Monte Carlo simulations, we cooled the system down to the temperatures where we studied nucleation: $T_{1}=800 \mathrm{~K}$ and $T_{2}=825 \mathrm{~K}$, corresponding to $25 \%$ and $22 \%$ undercooling. Note that the experiments on $\mathrm{NaCl}$ nucleation were performed at a somewhat higher temperature (16\% supercooling). The reason why we could not perform simulations at these higher temperatures is that the critical nucleus would be about twice the size of the nucleus that could be studied without spurious finite-size effects ${ }^{19}$ for the system sizes that we employed. At temperatures below $750 \mathrm{~K}$, spontaneous nucleation occurred during the simulations. We therefore kept the temperature above this lower limit.

Nucleation is an activated process. In steady state, the nucleation rate per unit volume and time is given by Eq. (3) (Refs. 2, 5, and 20) where $\exp \left(-\beta \Delta G_{\text {crit }}\right)$ is the equilibrium probability to find a critical nucleus in the metastable parent phase. $\kappa$ is a kinetic prefactor. In the case of a diffusive barrier crossing, $\kappa$ can be expressed as

$$
\kappa=\sqrt{\frac{|\Delta \mu|}{6 \pi k_{B} T n_{\text {crit }}}} \rho_{\text {liq }} f_{n_{\text {crit }}}^{+}
$$

where $\rho_{\text {liq }}$ is the ion density of the metastable liquid, $f_{n_{\text {crit }}}^{+}$the rate at which particles are added to a critical nucleus, $\Delta \mu$ $=\mu_{\mathrm{liq}}-\mu_{\mathrm{sol}}$ is the difference in chemical potential between liquid and solid and $\sqrt{|\Delta \mu| / 6 \pi k_{B} T n_{\text {crit }}}$ the Zeldovitch factor, that takes into account the fact that, during steady-state nucleation, the concentration of critical nuclei is, in fact, not the equilibrium concentration.

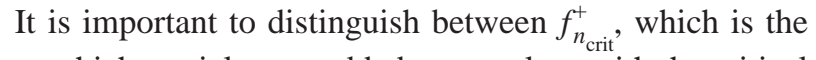
rate at which particles are added to a nucleus with the critical size, and the net flux across the nucleation barrier. In steady state, this net flux is equal to the number of nuclei that go from $n_{\text {crit }}$ to $n_{\text {crit }}+1$ minus the number that goes from $n_{\text {crit }}$ +1 to $n_{\text {crit }} \cdot{ }^{2-4}$ Hence, the actual nucleation rate is a combination of the forward rate $f^{+}$and the backward rate $f^{-}$. However, because of detailed balance, knowledge of $f_{n_{\text {crit }}^{+}}^{+}$(combined with knowledge of the barrier height and shape) is enough to compute the nucleation rate.

Assuming a diffusive attachment or detachment of single particles from the critical nucleus, the forward rate $f_{\text {crit }}^{+}$at the top of the barrier can be related to the spontaneous fluctuations in the number of particles in a nucleus at the top of the nucleation barrier:

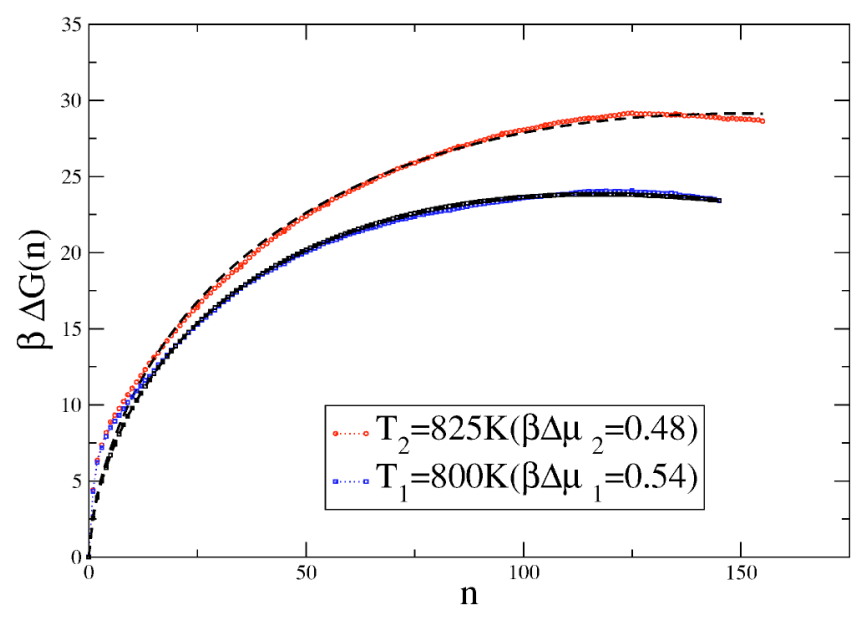

FIG. 1. Free-energy barriers $\Delta G$ as a function of the nucleus size $n$ for $T_{1}$ $=800 \mathrm{~K}\left(\beta \Delta \mu_{1}=0.54\right)$ and $T_{2}=825 \mathrm{~K}\left(\beta \Delta \mu_{2}=0.48\right)$. Error bars on $\beta \Delta G$ are of the order of $1 k_{B} T$. The dashed curves are fits to the functional form given by CNT.

$$
f_{\text {crit }}^{+}=\frac{1}{2} \frac{\left\langle\Delta n_{\text {crit }}^{2}(t)\right\rangle}{t}
$$

where $\left\langle\Delta n_{\text {crit }}^{2}(t)\right\rangle=\left\langle\left[n_{\text {crit }}(t)-n_{\text {crit }}(0)\right]^{2}\right\rangle$ is the mean-square change in the number of particles belonging to the critical nucleus during a time interval $t$. To estimate $f_{\text {crit }}^{+}$a series of dynamical trajectories was necessary; after generating a set of uncorrelated configurations at the top of the barrier, we carried out constant density and temperature MD simulations using the DL_POLY package ${ }^{21}$ with a time step of $0.5 \mathrm{fs}$. We computed the nucleation rate using Eq. (3).

Moreover, we computed the nucleation rate per unit time and volume using an algorithm based on path-sampling techniques ${ }^{14,15}$ and compared the results with those obtained using Eq. (3).

\section{RESULTS}

We computed the free-energy barrier for crystal nucleation at $T_{1}=800 \mathrm{~K}$ and $T_{2}=825 \mathrm{~K}$, corresponding to $\beta \Delta \mu_{1}$ $=0.54$ and $\beta \Delta \mu_{2}=0.48$. The values of $\beta \Delta \mu$ were estimated numerically by thermodynamic integration from the coexistence temperature and free energies reported by Anwar et $a l .{ }^{18}$ Figure 1 shows the computed nucleation barriers as a function of $n$.

As expected, $\Delta G$ decreases with supersaturation. Around $T<750 \mathrm{~K}$ the barrier gets sufficiently low that spontaneous nucleation can take place on the time scale of a simulation. The size of the critical nucleus, $N_{c}$, was estimated according to a fit of the functional form of the CNT, and we found $N_{c} \approx 120$ ions at $T_{1}$ and $N_{c} \approx 150$ ions at $T_{2}$. Koishi et al. ${ }^{9}$ estimated $N_{c}=120-130$ ions at 640 and $740 \mathrm{~K}$, which is surprising in view of the CNT prediction that the size of the critical nucleus scales as $(\gamma /|\Delta \mu|)^{3}$. If we make the usual assumption $^{5}$ that $\gamma \sim \Delta h$ and $\Delta \mu \approx\left(\Delta h / T_{m}\right)\left(T-T_{m}\right)$, where $\Delta h$ is the enthalpy of fusion per ion pair, then we would expect that $N_{c} \sim\left(1-T / T_{m}\right)^{-3}$ and we would predict that the critical nucleus at $640 \mathrm{~K}$ should contain a quarter as many particles as those at $800 \mathrm{~K}$. 


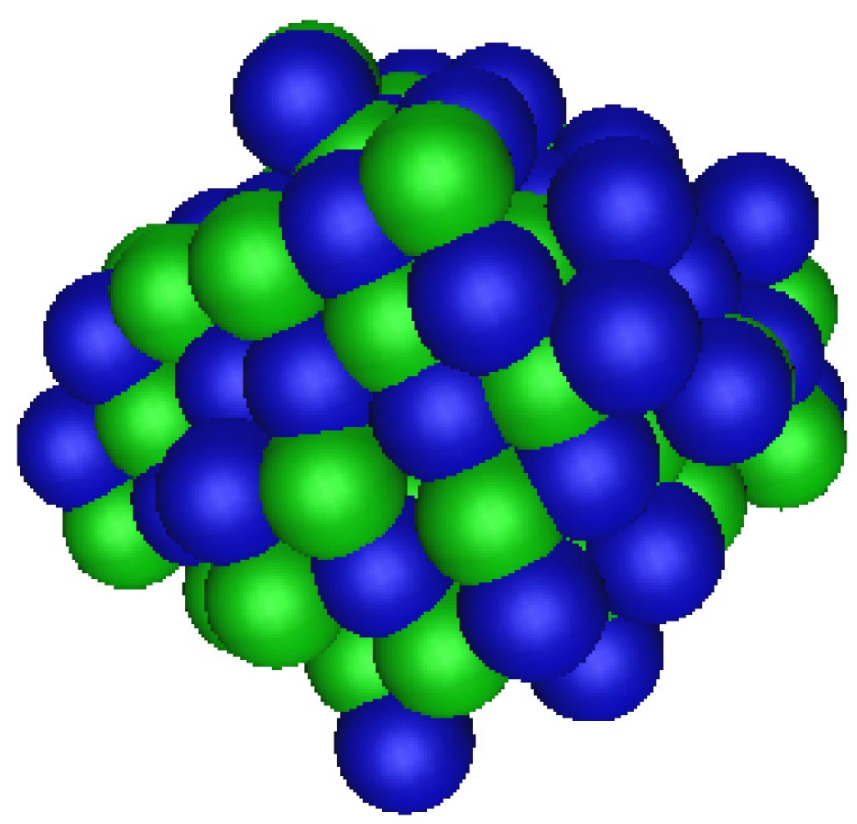

FIG. 2. Snapshot of the critical nucleus at $T_{2}=825 \mathrm{~K}$ : the bulk $\mathrm{NaCl}$ structure is already evident. The critical crystal nucleus seems to have rudimentary facets, which is in agreement with the interpretation of the experiments in Ref. 6.

Next, we consider the structure and shape of the critical nucleus.

Figure 2 shows a snapshot of the critical nucleus at $T_{2}$ $=825 \mathrm{~K}$. Note that the crystal presents rudimentary lowindex facets. In experiments ${ }^{6,22}$ the existence of such facets was postulated, as they may act as sites for subsequent heterogeneous nucleation.

As can be seen from Fig. 2, the critical nucleus already shows the charge-ordered rock-salt structure of the bulk phase. This indicates that, at least for $\mathrm{NaCl}$, nucleation does not proceed via an intermediate metastable phase. ${ }^{23}$ Figure 2 also shows that, in the temperature range that we studied, the critical nucleus is nonspherical. In order to quantify the degree of nonsphericity of the critical nucleus, we expanded its density with respect to the center of mass in rank-four spherical harmonics and constructed the quadratic invariant $S_{4}{ }^{24}$ We obtained $S_{4}\left(T_{1}\right)=0.115$ and $S_{4}\left(T_{2}\right)=0.110$. For a simple cube $S_{4}=0.172$ and for a sphere $S_{4}=0$, therefore the shape of the critical nucleus is closer to a cube than to a sphere. In other words, the critical nucleus already exhibits the morphology of macroscopic $\mathrm{NaCl}$ crystals. ${ }^{25}$

In order to make sure that there were no finite-size effects that resulted in interactions between image cluster, we visually checked that the critical nuclei did not show a preferred spacial orientation and that the minimum distance between them was bigger than a half box.

Moreover, we calculated the Debye-Huckle screening length and found that it was smaller than $1 \AA$, showing that the critical nuclei did not even electrostatically interact. We could therefore conclude that there was no induced nucleation due to the interaction between a critical cluster and its own periodic image.

Using the computed height of the nucleation barrier and the values of $\Delta \mu$ as input, we can estimate the surface free- energy density $\gamma_{l s}$. To this end, we make use of the CNT expression for the barrier height [Eq. (2)]. However, this expression assumes that the critical nucleus is spherical. It is easy to derive the corresponding expression for a cubical nucleus. The results for both estimates of $\gamma_{l s}$ are given in Table II. There exist experimental estimates of $\gamma_{l s}$ at $905 \mathrm{~K}^{6}$ These estimates are based on a somewhat questionable CNT expression for the nucleation rate. Moreover, in Ref. 6 it is assumed that the critical nucleus is spherical. The experimental estimate of $\gamma_{l s}\left(\gamma_{\exp }=84.1 \mathrm{erg} \mathrm{cm}^{-2}\right)$ is therefore not based on a direct determination. Nevertheless, in the absence of other experimental data, this is the only number we can compare to.

As the table shows, there is a fair agreement between simulation and experiment. The experimental estimate for $\gamma$ is based on the assumption that the critical nucleus is spherical. If it is cubic, one would obtain the number in the lower right-hand corner. In view of the many uncertainties in the analysis of the experimental data, it is impossible to tell whether the discrepancy between simulation and experiment is significant. An estimate of $\gamma$ based on the experimental enthalpy of fusion following Turnbull, would yield $\gamma \approx 115 \mathrm{erg} \mathrm{cm}^{-2}$ (see, e.g., Ref. 8).

Huang et $a .^{8}{ }^{8}$ estimated the solid-liquid surface freeenergy density of $\mathrm{NaCl}$ from the nucleation rate at $550 \mathrm{~K}$. To achieve this, Huang et al. assumed that the CNT expression for the nucleation rate is valid. Under those assumptions, they obtained $\gamma=119.6 \mathrm{erg} \mathrm{cm}^{-2}$ for a spherical nucleus.

In the present work, we can compute absolute nucleation rates without making use of CNT. The only assumption we make is that the barrier crossing is diffusive and that the Zeldovitch (pre)factor is well approximated by the form given in Eq. (4). The Zeldovitch factors were found to be, respectively, $Z_{1}=0.016$ for $T_{1}$ and $Z_{2}=0.013$ for $T_{2}$. The true Zeldovitch factor may be slightly different, but is in any event expected to be of $\mathcal{O}\left(10^{-2}\right)$. From our MD simulations we obtained the following estimates for the forward rates [Eq. (7)]: $f_{\text {crit }}^{+}=0.013 \mathrm{ps}^{-1}$ for $T_{1}$ and $f_{\text {crit }}^{+}=0.033 \mathrm{ps}^{-1}$ for $T_{2}$. Combining this information, we can compute the kinetic prefactor of Eq. (6): $\kappa\left(T_{1}\right)=6.9 \times 10^{36} \mathrm{~m}^{-3} \mathrm{~s}^{-1}$ and $\kappa\left(T_{2}\right)$ $=1.5 \times 10^{37} \mathrm{~m}^{-3} \mathrm{~s}^{-1}$. As is to be expected, the kinetic prefactor depends only weakly on temperature. Using Eq. (4) we then calculated the nucleation rate. The results are $I\left(T_{1}\right) O\left(10^{26 \pm 1}\right) \mathrm{m}^{-3} \mathrm{~s}^{-1}$ and $I\left(T_{2}\right) O\left(10^{24 \pm 1}\right) \mathrm{m}^{-3} \mathrm{~s}^{-1}$.

These nucleation rates are about ten orders of magnitude higher than the estimated experimental rate at $905 \mathrm{~K}$ $\left[O\left(10^{16}\right) \mathrm{m}^{-3} \mathrm{~s}^{-1}\right]$. Such a difference is hardly surprising because the nucleation rate is expected to increase rapidly with increasing supercooling.

We also computed the nucleation rate using an algorithm based on the path-sampling techniques of Refs. 14 and 15 (FFS and TIS). The value obtained at $T_{1}=800 \mathrm{~K}$ is $I_{\text {FFS-TIS }}\left(T_{1}\right)=O\left(10^{27 \pm 2}\right) \mathrm{m}^{-3} \mathrm{~s}^{-1}$, which agrees surprisingly well with the one obtained using the diffusive barriercrossing approach.

As the path-sampling method does not depend on the choice of the reaction coordinate and does not require prior knowledge of the phase-space density, we can conclude that 
TABLE III. Summary of the simulation results for the calculation of the free-energy barrier and the nucleation rate for Tosi-Fumi $\mathrm{NaCl}$.

\begin{tabular}{cccccc}
\hline \hline$T(\mathrm{~K})$ & $\beta \Delta \mu$ & $\beta \Delta G_{\text {crit }}$ & $f_{\text {ncrit }}^{+}$ & $I\left(\mathrm{~m}^{-3} \mathrm{~s}^{-1}\right)$ & $I_{\text {FFS-TIS }}\left(\mathrm{m}^{-3} \mathrm{~s}^{-1}\right)$ \\
\hline 800 & 0.54 & 24 & 0.013 & $O\left(10^{26 \pm 1}\right)$ & $10^{27 \pm 2}$ \\
825 & 0.48 & 29 & 0.033 & $O\left(10^{24 \pm 1}\right)$ & $\cdots$ \\
\hline \hline
\end{tabular}

the method based on the free-energy calculation gives us a good estimate for the nucleation rate at this temperature.

We have also computed the nucleation rate at $T$ $=750 \mathrm{~K}$, using the FFS-TIS method. The computed nucleation rate is $I_{\mathrm{FFS}-\mathrm{TIS}}(T=740 \mathrm{~K})=O\left(10^{35}\right) \mathrm{m}^{-3} \mathrm{~s}^{-1}$. This is of the same order of magnitude as the nucleation rate obtained calculated by Koishi et al. ${ }^{9}$ using "brute force" MD. We cannot use the diffusive barrier crossing method at this temperature, as the barrier is too low to avoid spontaneous nucleation during long runs. However, if we assume that the kinetic prefactor and the surface free-energy density do not vary much with temperature, we can use CNT to extrapolate the nucleation rate from 800 to $740 \mathrm{~K}$. We find $I_{\text {extrap }}(T$ $=740 \mathrm{~K})=O\left(10^{30}\right) \mathrm{m}^{-3} \mathrm{~s}^{-1}$ which is considerably lower than the results of the direct calculations. This suggests that an extrapolation procedure based on CNT is not reliable. A summary of our numerical results for the nucleation barriers and rates is given in Table III.

A similar problem occurs if we try to extrapolate our numerical data at 800 and $825 \mathrm{~K}$ to $905 \mathrm{~K}$, the temperature of the experiments of Ref. 6. If we can extrapolate our simulation results to the experimental temperature of $905 \mathrm{~K}$ $(\beta \Delta \mu=0.3)$ we obtain an estimated nucleation at $905 \mathrm{~K}$ that is $O\left(6 \times 10^{11}\right) \mathrm{m}^{-3} \mathrm{~s}^{-1}$. This is some nearly five orders of magnitude less than the experimentally observed rate.

The discrepancy between simulation and experiment can be due to several reasons. (a) There might be an appreciable (but unspecified) error in the experimental estimates (e.g., due to residual heterogeneous nucleation). (b) The estimated error in the computed melting temperature of the Tosi-Fumi model is $\pm 20 \mathrm{~K} .{ }^{18}$ Such an uncertainty again easily translates into a variation of the nucleation rate by several orders of magnitude. (c) In view of the extreme sensitivity of nucleation rates to the details of the intermolecular potential (see, e.g., Ref. 26), the Tosi-Fumi potential may be inadequate to model nucleation in $\mathrm{NaCl}$, even though it can reproduce the static properties of the solid and liquid $\mathrm{NaCl}^{27}$ (d) Finally, it is not quite correct to assume that the kinetic prefactor, the surface free energy, and the latent heat of fusion are temperature independent.

We can also compare our calculated nucleation rate at $800 \mathrm{~K}$ to the rate estimated with $\mathrm{CNT}$. In order to do that, we need to compute the kinetic prefactor $\kappa_{\mathrm{CNT}}$ that, using the CNT approximations, is ${ }^{5}$

$$
\kappa_{\mathrm{CNT}}=Z \rho_{\text {liq }} \frac{24 D_{S} n_{\mathrm{crit}}^{2 / 3}}{\lambda^{2}} \text {. }
$$

The attachment rate of particles to the critical nucleus $\left(f_{n_{\text {crit }}}^{+}\right)$ takes into account the number of available attachment sites on the surface of a spherical nucleus $\left(n_{\text {crit }}^{2 / 3}\right)$ and depends on the jump frequency for bulk diffusion $\left(D_{S} / \lambda^{2}\right)$, where $\lambda$ is the atomic jump distance. Since the functional form of the nucleation barrier can be fitted to the corresponding CNT expression, the computed Zeldovitch factor $(Z)$ coincides with the predicted one. We computed the self-diffusion coefficient with MD simulations using the DL_POLY package ${ }^{21}$ in the supercooled liquid at $T_{1}=800 \mathrm{~K}$. We found $D_{S}^{\mathrm{Na}}=3.4$ $\times 10^{-5} \mathrm{~cm}^{2} \mathrm{~s}^{-1}$ in good agreement with an estimate $\left(D_{S}^{\mathrm{Na}}\right.$ $=2.3 \times 10^{-5} \mathrm{~cm}^{2} \mathrm{~s}^{-1}$ ) based on extrapolation of the available experimental data of Ref. 28 to the temperature $T_{1}$. Since $D_{S}^{\mathrm{Na}} / D_{S}^{\mathrm{Cl}} \approx 1$, we only considered the self-diffusion of the $\mathrm{Na}^{+}$ions. We estimated $\lambda$ as a fitting parameter from the $f_{n_{\text {crit }}^{+}}$ previously calculated; we obtained $\lambda\left(T_{1}\right)=10^{2} \AA$. However, considering that the ion size is $\sigma_{\mathrm{Na}} \sim 1.1 \AA$, this value for the jump distance seems unphysical $(\lambda \sim 100 \sigma)$. Typically, one would expect $\lambda$ to be of the order of a mean free path. In a molten salt, the mean free path of an ion is certainly less than a particle diameter. This discrepancy also suggests that the CNT picture is inadequate to describe crystal nucleation of $\mathrm{NaCl}$.

In summary, we have computed the crystal nucleation rate of sodium chloride from the melt using two independent methods: one based on the calculations of the free-energy barrier and the diffusive barrier crossing and another based on a path-sampling approach. We have found that, within an order of magnitude, the two approaches yield the same value for the nucleation rate. When we use classical nucleation theory to extrapolate our numerical data to lower temperatures, we observe serious discrepancies with the results of direct calculations. When we use CNT to extrapolate to high temperatures, we find serious discrepancies with the nucleation rates found in experiments. Several factors may contribute to this discrepancy but, at present, it is not yet known which factor is most important.

\section{ACKNOWLEDGMENTS}

The work of the FOM Institute is part of the research program of FOM and is made possible by financial support from the Netherlands Organization for Scientific Research (NWO). One of the authors (C.V.) gratefully acknowledges the financial support provided through the European Community Human Potential Program under Contract No. HPRN-CT-1999-00025, (Nucleus). Another author (E.S.) gratefully acknowledges the Spanish government for the award of a FPU Ph.D. grant, and the FOM Institute for the hospitality during the period in which this work was carried out. Two of the authors (C.V. and E.S.) thank Angelo Cacciuto and Rosalind Allen for valuable discussions and suggestions, and Georgios Boulougouris and Josep Pámies for a critical reading of the manuscript. 


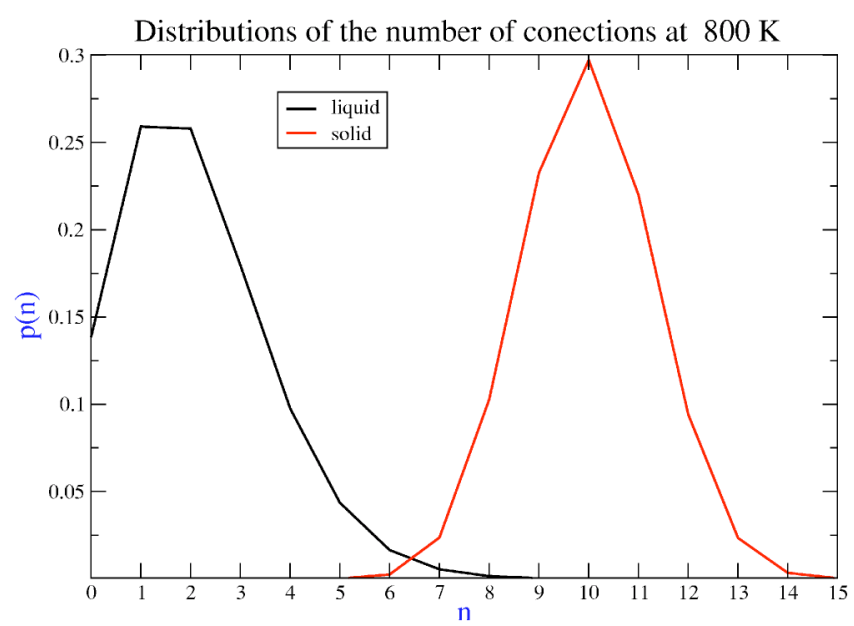

FIG. 3. Distributions of the number of scalar products bigger than 0.35 at $T=800 \mathrm{~K}$ for the solid and for the liquid.

\section{APPENDIX: IDENTIFICATION OF CRYSTALLINE CLUSTERS}

To distinguish between solid and liquidlike particles and identify the particles belonging to a solid cluster, we used the local bond order parameter introduced by ten Wolde et al. ${ }^{29}$ Although the method we used is the same as the one proposed by ten Wolde, the definition of a "solidlike" particle is not rigorously the same. The $q$ vector and the thresholds selected were optimized for the $\mathrm{NaCl}$ Tosi-Fumi model.

First, we computed a normalized complex vector $q_{4}$ for every particle $i$. Each component of this vector was given by

$$
\mathbf{q}_{4, m}(i)=\frac{\frac{1}{N_{b}(i)} \sum_{j}^{N_{b}(i)} \Upsilon_{4, m}\left(\theta_{i, j}, \phi_{i, j}\right)}{\mathbf{q}_{4, m}(i) \cdot \mathbf{q}_{4, m}^{*}(i)}, \quad m=[-4,4],
$$

where $N_{b}(i)$ is the number of neighbors of the particle $i$ within a cut-off radius of $4 \AA$ (the first minimum in the $\mathrm{Na}-\mathrm{Cl}$ radial distribution function).

Then we computed a scalar product $q_{4}(i) \cdot q_{4}^{*}(j)$ for every particle $i$ with each of its neighbors particle $j$. A particle was considered to be solidlike when at least six of the scalar products were bigger than 0.35. Finally, two solidlike particles were considered to be neighbors in the same cluster if they were closer than $3.4 \AA$.
Ionic fluids are more ordered than Lennard-Jones or hard spheres ones, as the radial distribution function shows. However, with the method implemented by ten Wolde, we were able to clearly distinguish between solid and liquidlike particles in the $\mathrm{NaCl}$ Tosi-Fumi model. We enclose a plot (Fig. 3 ) that shows the distributions of the number of scalar products bigger than 0.35 at $T=800 \mathrm{~K}$ for the solid and for the liquid; for values bigger than six a particle was considered to be solidlike.

${ }^{1}$ J. W. Gibbs, The Scientific Papers of J. Willard Gibbs (Dover, New York, 1961).

${ }^{2}$ M. Volmer and A. Weber, Z. Phys. Chem. 119, 227 (1926).

${ }^{3}$ L. Farkas, Z. Phys. Chem. 125, 236 (1927).

${ }^{4}$ R. Becker and W. Döring, Ann. Phys. 24, 719 (1935).

${ }^{5}$ K. F. Kelton, Crystal Nucleation in Liquids and Glasses (Academic, Boston, 1991), Vol. 45, pp. 75-177.

${ }^{6}$ E. R. Buckle and A. R. Ubbelohde, Proc. R. Soc. London, Ser. A 529, 325 (1960); 261, 197 (1961).

${ }^{7}$ E. R. Buckle, Proc. R. Soc. London, Ser. A 261, 189 (1961).

${ }^{8}$ J. Huang, X. Zhu, and L. S. Bartell, J. Phys. Chem. A 102, 2708 (1998).

${ }^{9}$ T. Koishi, K. Yasuoka, and T. Ebisuzaki, J. Chem. Phys. 119, 11298 (2003).

${ }^{10}$ J. S. Van Duijneveldt and D. Frenkel, J. Chem. Phys. 96, 4655 (1992).

${ }^{11}$ P. R. ten Wolde and D. Frenkel, J. Chem. Phys. 109, 9901 (1998).

${ }^{12}$ S. Auer and D. Frenkel, J. Chem. Phys. 120, 3015 (2004).

${ }^{13}$ G. M. Torrie and J. P. Valleau, Chem. Phys. Lett. 28, 578 (1974).

${ }^{14}$ R. J. Allen, P. B. Warren, and P. R. ten Wolde, Phys. Rev. Lett. 94, 018104 (2005).

${ }^{15}$ T. S. Van Erp, D. Moroni, and P. G. Bolhuis, J. Chem. Phys. 118, 7762 (2003).

${ }^{16}$ F. Fumi and M. Tosi, J. Phys. Chem. Solids 25, 31 (1964), M. Tosi and F. Fumi, ibid. 25, 45 (1964).

${ }^{17}$ G. J. Janz, Molten Salt Handbook (Academic, New York, 1967).

${ }^{18}$ J. Anwar, D. Frenkel, and M. Noro, J. Chem. Phys. 188, 728 (2003).

${ }^{19}$ J. D. Honeycutt and H. C. Andersen, Chem. Phys. Lett. 108, 535 (1984).

${ }^{20}$ D. W. Oxtoby, J. Phys.: Condens. Matter 4, 7627 (1992).

${ }^{21}$ W. Smith and T. Forester, J. Mol. Graphics 14, 136 (1996).

${ }^{22}$ P. Balk and G. C. Benson, J. Phys. Chem. 63, 1009 (1959).

${ }^{23}$ W. Ostwald, Z. Phys. Chem., Stoechiom. Verwandtschaftsl. 22, 289 (1897).

${ }^{24}$ P. R. ten Wolde, M. J. Ruiz-Montero, and D. Frenkel, Phys. Rev. Lett. 75, 2714 (1995).

${ }^{25}$ J. C. Heyraud and J. J. Métois, J. Cryst. Growth 82, 269 (1987).

${ }^{26}$ S. Auer, W. C. K. Poon, and D. Frenkel, Phys. Rev. E 67, 020401 (2003).

${ }^{27}$ T. Zykova-Timan, U. Tartaglino, D. Ceresoli, and E. Tosatti, Surf. Sci. 566, 794 (2004).

${ }^{28}$ J. O' M. Bockris, S. R. Richards, and L. Nanis, J. Phys. Chem. 69, 1627 (1965).

${ }^{29}$ P. R. ten Wolde, M. J. Ruiz-Montero, and D. Frenkel, Faraday Discuss. 104, 93 (1996). 